\title{
Stabilization with arbitrary laser polarizations
}

\author{
A. Patel, ${ }^{1}$ M. Protopapas, ${ }^{1}$ D. G. Lappas, ${ }^{2}$ and P. L. Knight ${ }^{1}$ \\ ${ }^{1}$ Optics Section, Blackett Laboratory, Imperial College London, London SW7 2BZ, United Kingdom \\ ${ }^{2}$ Department of Physics, Lund Institute of Technology, P.O. Box 118, S-221 00 Lund, Sweden
}

(Received 13 March 1998; revised manuscript received 9 June 1998)

\begin{abstract}
The interaction of an atom with a very strong and high-frequency laser is investigated in the context of electron wave-packet stabilization and localization. We employ an exact numerical solution of the Schrödinger equation in two space dimensions, which allows for an arbitrary laser ellipticity. Substantially different degrees of stabilization and electron localization patterns are found for various ellipticities. Circular polarization leads to the highest degree of stabilization. [S1050-2947(98)50210-7]
\end{abstract}

PACS number(s): 42.50.Hz, 32.80.Fb, 32.80.Wr

The nonperturbative interaction of intense laser light with atoms has been an area of very active research for many years [1]. One of the most surprising findings has been stabilization, the reduction in ionization as a function of increasing intensity in the interaction of atomic systems with intense high-frequency laser fields. This was initially discovered by Gersten and Mittleman [2], and described quantitatively by Gavrila [3] using the time-independent highfrequency Floquet theory in the Kramers-Henneberger (KH) frame. They were able to calculate ionization rates and predict the forms of the stabilized wave packets. Timedependent results were presented by $\mathrm{Su}$ and co-workers for the one-dimensional Schrödinger equation [4], and in three dimensions by Kulander et al. [5], which confirmed the possibility of stabilization in pulsed laser fields given optimal turn-on times.

In all time-dependent studies to date, the interaction has been driven by a linearly polarized laser field. The reason for this restriction has been the prohibitively large basis set required to adequately describe time-dependent interactions with elliptically polarized light. Recently, we have proposed and implemented a method for numerically solving the twodimensional Schrödinger equation, allowing the investigation of laser-atom interactions with arbitrary laser polarizations, in which the only approximations are the reduced dimensionality and the smoothing of the Coulomb singularity [6]. In this Rapid Communication we use this approach to investigate time-dependent stabilization in high-intensity and high-frequency fields of arbitrary polarization. We present results of the dependence of stabilization on ellipticity and predict unusual toroidal stabilized wave packets. The survival of a stabilized wave packet, driven by a pulsed laser field with circular polarization, has not been investigated previously. Whether those stabilized features predicted by adiabatic Floquet methods [3] survive pulse turn-on effects is the subject of the present Rapid Communication.

The two-dimensional Schrodinger equation that we solve, in atomic units (a.u.), is of the form

$$
\begin{aligned}
\mathrm{i} \frac{\partial}{\partial t} \Psi(x, y ; t)= & -\frac{1}{2}\left(\frac{\partial^{2}}{\partial x^{2}}+\frac{\partial^{2}}{\partial y^{2}}\right)-\frac{1}{\sqrt{a^{2}+x^{2}+y^{2}}} \\
& +(x E \sin \omega t+\epsilon y E \cos \omega t) f(t)] \Psi(x, y ; t),
\end{aligned}
$$

where $\Psi(x, y ; t)$ is the two-dimensional wave function, $f(t)$ is the pulse envelope, and $\epsilon$ is the polarization ellipticity. The second term on the right-hand side is the smoothed Coulomb potential [4] used to avoid numerical problems associated with the singularity at $x=y=0 \quad(a=0.8$, so that the ground state has the same binding energy as hydrogen, i.e., -0.5 a.u.). The pulse shape $f(t)$ is trapezoidal, with twocycle turn-on times, followed by ten cycles of constant amplitude, and then a two-cycle turn-off time. This pulse shape ensures no drift motion in the free-electron displacement at the end of the pulse. However, during the pulse, there is a small drift of the wave packet around the origin, which is of the order $E / \omega^{2}$ along the $y$ axis, at the time of our wave packet snapshots. This drift will be evident in the wave packets that are presented below.

Stabilization depends strongly on the pulse turn-on cycle $[5,7,8]$; we have found two cycles to be short enough to ensure that enough of the population survives the turn-on yet long enough so that the population is not immediately shock excited into the continuum. We have also chosen to use a high frequency, $\omega=1.0$ a.u., which guarantees that the conditions necessary for stabilization are easily satisfied. Since the numerical method utilizes an absorbing boundary to remove any ionized wave packet reaching the integration grid edge, the normalization of the evolving wave packet can be used as a measure of the occurring ionization. A more detailed account of the various numerical aspects can be found in Refs. [6, 9].

Much of the interpretation and understanding of the physics involved in stabilization depends on the form of the stabilized wave packets. It is therefore informative initially to examine the following. In Fig. 1 we present the wave packet $|\psi(x, y)|^{2}$, generated after 12 cycles of our pulse, i.e., just before the turn-off. In Fig. 1(a) we show the wave packet that is produced by linearly polarized light; we find similar results to those of Kulander et al. [5], with a wave packet made up of two large peaks separated by $2 \alpha_{0}$, where $\alpha_{0}$ is the maximum classical displacement of an electron in the incident field $\left(\alpha_{0}=E / \omega^{2}\right)$. A third, generally less localized peak placed centrally between these two larger peaks, is also observed. In Fig. 1(b) we show the wave packet generated by an elliptically polarized $E$ field, with $E=15$ a.u. at the peak of the major axis. The intensity here is given by $I=E^{2}(1$ $+\epsilon^{2}$ ), with $\epsilon=0.5$. Along the $x$ axis, we again find two very localized peaks separated by $2 \alpha_{0}$, while along the $y$ axis we 

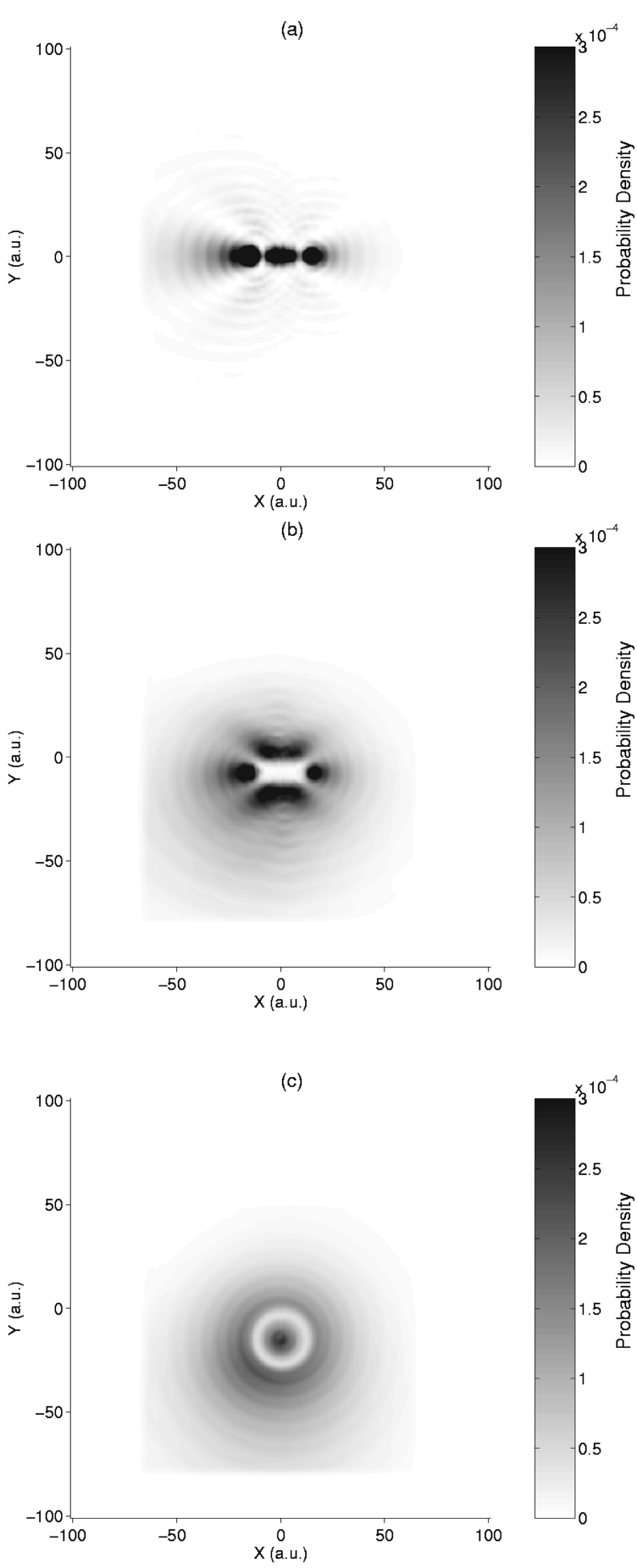

FIG. 1. (a) Snapshot of the wave packet after 12 cycles of a laser pulse with frequency $\omega=1$ a.u., and peak electric field $E$ $=15$ a.u. with linear polarization; (b) ellipticity $\epsilon=0.5$. (c) ellipticity $\epsilon=1$. Distances $(X, Y)$ are shown in atomic units (a.u.).

find two "kidney"'-shaped localizations separated by $\epsilon 2 \alpha_{0}$. We also note the absence of wave-packet density between these peaks; there is almost no probability of finding the packet in this region. The explanation of this extraordinary wave packet lies in the cycle-averaged $\mathrm{KH}$ potential [3]. In the $\mathrm{KH}$ frame it is the nuclear potential that oscillates. If this oscillation is fast, an averaged potential may be used that has minima at the turning points. Around those minima the wave packet will localize. In Fig. 2 we show the cycle-averaged potential for the parameters of Fig. 1(b). The potential is

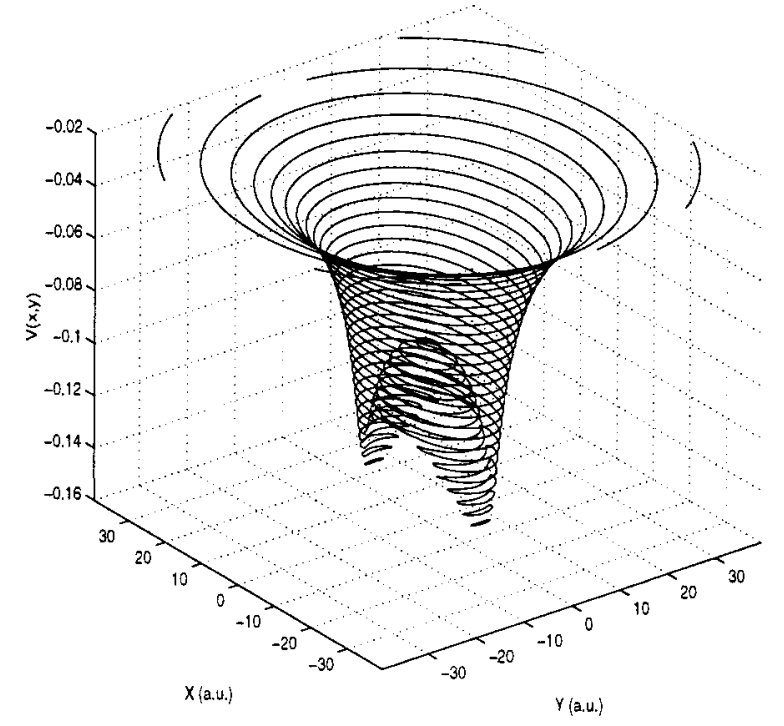

FIG. 2. The cycle-averaged Kramers-Henneberger potential $V(x, y)$, in atomic units, for the parameters of Fig. 1(b).

negative, with varying depth along the elliptical ring. Two minima lie along the $x$ axis at $\pm \alpha_{0}$, about which we would expect localization. However, in addition, there are two saddle points at $\pm \epsilon \alpha_{0}$ along the $y$ axis. From a comparison with the KH ground state (not presented here) we have concluded that the kidney-shaped localizations shown in Fig. 1(b) are due to the interferences between higher $\mathrm{KH}$ eigenstates. In Fig. 1(c) we show the stabilized wave packet generated by circularly polarized light of the peak field $E$ $=15$ a.u. In this case, one would expect the cycle-averaged $\mathrm{KH}$ potential to be a circular trough of radius $\alpha_{0}$ around which the wave packet would stabilize. This is precisely what is seen: a toroidal "ring" wave packet is formed with a radius that is approximately 15 a.u. (this is in agreement with the prediction from the time-independent approaches of Pont and Gavrila [10]). In addition we also find a large peak at the center of this circle. This central peak occurs because of the nonadiabaticity of the pulse, which, as in the elliptical case, creates a superposition of cycle-averaged $\mathrm{KH}$ potential states $[5,7,11,12]$. As for the elliptical wave packet, we find a region of essentially zero wave-packet density between the ring and the central peak.

The signature of stabilization is a decrease in ionization as intensity increases. In Fig. 3 we plot the normalization at the end of the pulse versus the peak intensity $I=E^{2}\left(1+\epsilon^{2}\right)$. Initially, as the intensity is increased a fast drop in the normalization is seen, with a dependence on the ellipticity that can be understood to be due to the geometry of the interaction [6]. Eventually a minimum is reached, after which the normalization becomes an increasing function of intensity as stabilization sets in. The minimum in the normalization shifts to higher intensities with increasing ellipticity. We find the following values for the critical electron displacement amplitude $\alpha_{c x}^{\epsilon}$ along the $x$ axis: $\alpha_{c x}^{0}=1.95$ a.u., $\alpha_{c x}^{0.5}=1.88$ a.u., and $\alpha_{c x}^{1}=1.64$ a.u. This shows a clear decrease of the $\alpha_{c x}^{\epsilon}$ for stabilization with increasing ellipticity. A simple intuitive understanding of this observation is that when the electron wave packet misses the atomic core, increasingly with higher ellipticity, the cycle-averaged interaction strength with the 


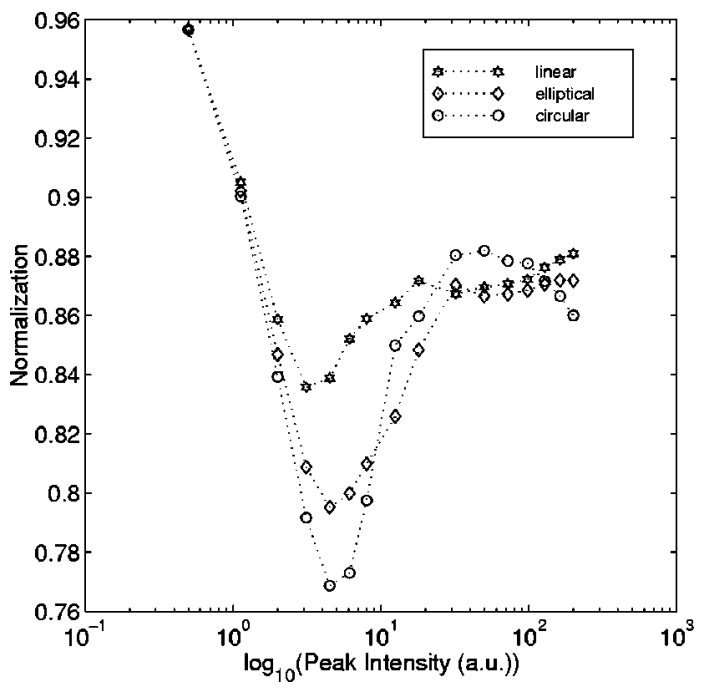

FIG. 3. Normalization as a function of peak laser intensity $I=E^{2}\left(1+\epsilon^{2}\right)$ for a 14-cycle pulse with $\omega=1$ a.u., and ellipticities $\epsilon=0,0.5$, and 1 .

atomic core decreases. We shall see below that this fact is reflected in the values of the binding energies of the timeaveraged $\mathrm{KH}$ potential for different ellipticities. At intensities greater than 10 a.u. we find small oscillations in the normalization. These oscillations have been previously observed in stabilization studies with linearly polarized light, and have been assigned to the nonadiabaticity of the laser interaction $[5,8,13,14]$. A major implication drawn from this graph is that stabilization is greatest for circular polarization, defined in terms of the depth of the minima in relation to the point at which the oscillations occur. The most influential stabilization condition is $\omega \gg\left|I_{k h}^{\epsilon}\right|$, where $\left|I_{k h}^{\epsilon}\right|$ is the binding energy of the cycle-averaged $\mathrm{KH}$ potential [3]. For a given $E$ field of $E=15$ a.u. at its peak, we find $\left|I_{k h}^{0}\right| \approx 0.25>\left|I_{k h}^{0.5}\right|$ $\approx 0.15>\left|I_{k h}^{1}\right| \approx 0.10$, implying that circular polarization should stabilize to a greater degree, as we observe. Also, on physical grounds, one can see that there is less ionization (greater stabilization) for the circular case than for the linear case because the wave packet never passes through the nucleus. In contrast, in the linear case it scatters through the origin during the turn-on cycle. The somewhat different requirements for stabilization in the case of a circularly polarized laser could make the experimental observation of stabilization easier. However, prior to that, a more rigorous experimental investigation of angle-resolved ionization for circular polarization is needed.

In Fig. 4 we plot the harmonics generated at an intensity of $I=4.5$ a.u. for the three ellipticities considered. We find that the harmonics depend similarly on ellipticity as in other interaction regimes, with linear polarization being the most efficient and circular polarization the least efficient. This may seem surprising since the reocurring collisions that are needed to generate high harmonics do not occur efficiently in the stabilization regime. The explanation for the dependence of these harmonics on ellipticity is derived from the structure arguments proposed in Refs. $[15,16]$. The dipole acceleration, from which the harmonics are computed, can be calculated in the $\mathrm{KH}$ frame:

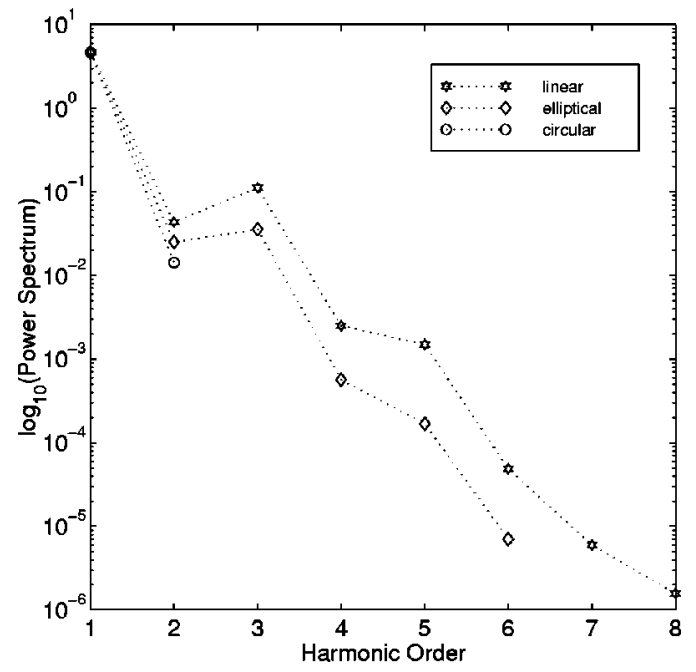

FIG. 4. Harmonic spectra for a 14-cycle laser pulse of peak intensity $I=4.5$ a.u., frequency $\omega=1$ a.u., and ellipticities $\epsilon=0$, 0.5 , and 1 .

$$
\ddot{\boldsymbol{d}}(t)=\left\langle\Psi_{k h}\left|\frac{r+\boldsymbol{\alpha}(t)}{\left[a^{2}+(\boldsymbol{r}+\boldsymbol{\alpha}(t))^{2}\right]^{3 / 2}}\right| \Psi_{k h}\right\rangle,
$$

where $\Psi_{k h}$ is the wave function in the KH frame and $\boldsymbol{\alpha}(t)$ is the classical electron displacement in the incident electric field. The $\ddot{\boldsymbol{d}}(t)$ operator resembles a derivative function, which then moves along the trajectory $\boldsymbol{\alpha}(t)$. This means that any wave packet that has many sharp features along $\boldsymbol{\alpha}(t)$ will produce high-frequency components within $\ddot{\boldsymbol{d}}(t)$ and, therefore, many harmonics. Returning to Fig. 1 and comparing the structure in the ellipse for case (b) and the circle for case (c), one can see that there are significantly more sharp features in the former case (b). Consequently, as is observed, more harmonics are generated by elliptically than by circularly polarized light. In the linear case we find a tridentshaped wave packet with large sharp features, which therefore produces the most efficient harmonic generation. Also, we still see a few harmonics even for circular polarization; this is because the density along the ring of the stabilized wave packet is not uniform due to the instantaneous laser field direction and the nonadiabaticity of the pulse. We also observe odd and even harmonics for all ellipticities, since the eigenfunctions of the cycle-averaged $\mathrm{KH}$ potential are not parity eigenstates in the laboratory frame $[5,15,17]$.

In conclusion, we have found stabilization in timedependent laser fields with arbitrary polarizations. We demonstrated that toroidal stabilized wave-packet shaping can be generated in time-dependent fields. We have also found that circular polarization is the most favorable for electron stabilization and localization. Also, harmonic generation depends on ellipticity in a similar way as in the well-studied regime of low laser frequency.

This work has been funded in part by the U.K. Engineering and Physical Sciences Research Council and the European Union. One of us (D.G.L.) acknowledges support through the EU Training and Mobility of Researchers Scheme. 
[1] For a recent review see, e.g., M. Protopapas, C. H. Keitel, and P. L. Knight, Rep. Prog. Phys. 60, 389 (1997).

[2] J. Gersten and M. H. Mittleman, J. Phys. B 9, 2561 (1976).

[3] For a review of this work see, e.g., M. Gavrila, in Atoms in Intense Laser Fields, edited by M. Gavrila (Academic, London, 1992), p. 435.

[4] Q. Su, J. H. Eberly, and J. Javanainen, Phys. Rev. Lett. 64, 862 (1990); Q. Su and J. H. Eberly, Phys. Rev. A 43, 2474 (1991); see also J. H. Eberly, R. Grobe, C. K. Law, and Q. Su, in Atoms in Intense Laser Fields (Ref. [3]), p. 301.

[5] K. C. Kulander, K. J. Schafer, and J. L. Krause, Phys. Rev. Lett. 66, 2601 (1991); K. C. Kulander, K. J. Schafer, and J. L. Krause, in Atoms in Intense Laser Fields (Ref. [3]), p. 247.

[6] M. Protopapas, D. G. Lappas, and P. L. Knight, Phys. Rev. Lett. 79, 4550 (1997); see also T. Zuo and A. N. Bandrauk, J. Nonlinear Opt. Phys. Mater. 4, 533 (1995).

[7] R. M. A. Vivirito and P. L. Knight, J. Phys. B 28, 4357 (1995).
[8] Q. Su, B. P. Irving, C. W. Johnson, and J. H. Eberly, J. Phys. B 29, 5755 (1996).

[9] For a more technical discussion on the two-dimensional Schrödinger equation, see R. Grobe and J. H. Eberly, Phys. Rev. A 48, 4664 (1993).

[10] M. Pont, Phys. Rev. A 40, 5659 (1989); see also Ref. [3].

[11] V. C. Reed, P. L. Knight, and K. Burnett, Phys. Rev. Lett. 67, 1415 (1991).

[12] J. C. Wells, I Simbotin, and M. Gavrila, Phys. Rev. A 56, 3961 (1997).

[13] G. Yao and S.-I. Chu, Phys. Rev. A 45, 6735 (1992).

[14] T. Millack, J. Phys. B 26, 4777 (1993).

[15] M. Protopapas, P. L. Knight, and K. Burnett, Phys. Rev. A 49, 1945 (1994).

[16] M. Protopapas, D. G. Lappas, C. H. Keitel, and P. L. Knight, Phys. Rev. A 53, R2933 (1996).

[17] M. H. Mittleman, Phys. Rev. A 46, 4209 (1992). 